14.1

\title{
Наноразмерный корреляционный анализ морфологической, оптической и магнитной структуры полимерных микросфер для мультиплексной диагностики
}

\author{
() К.Е. Мочалов ${ }^{1}$, О.И. Агапова ${ }^{2}$, А.Н. Генералова ${ }^{1}$, И.С. Васкан ${ }^{1,3}$, Д.О. Соловьева ${ }^{1}$, В.А. Олейников ${ }^{1}$, \\ И.И. Агапов ${ }^{2}$, А.Е. Ефимов $2,4,9$ \\ ${ }^{1}$ Институт биоорганической химии им. академиков М.М. Шемякина и Ю.А. Овчинникова РАН, Москва, Россия \\ ${ }^{2}$ Национальный медицинский исследовательский центр трансплантологии и искусственных органов \\ им. акад. В.И. Шумакова Минздрава России, Москва, Россия \\ ${ }^{3}$ Московский физико-технический институт (Национальный исследовательский университет), Долгопрудный, Московская \\ обл., Россия \\ ${ }^{4}$ OОО „СНОТРА“, Москва, Россия \\ ฯ E-mail: antefimov@gmail.com
}

Поступило в Редакцию 7 ноября 2019 г.

В окончательной редакции 28 ноября 2019 г.

Принято к публикации 28 ноября 2019 г.

\begin{abstract}
Представлена единая аналитическая технология, основанная на интеграции сканирующей зондовой нанотомографии и оптической микроскопии $(\mathrm{C} 3 \mathrm{HT}-\mathrm{OM})$ и продемонстрирован потенциал технологии на примере многопараметрического анализа кодируемых микросфер. Представленная технология СЗНТ-ОМ может стать мощным инструментом для мультимодальной нанохарактеризации широкого спектра микрочастиц, композитных и гибридных полимеров.
\end{abstract}

Ключевые слова: мультиплексный анализ, наночастицы, корреляционная нанотомография, флуоресцентная спектроскопия, магнитно-силовая микроскопия.

DOI: 10.21883/PJTF.2020.05.49103.18103

За последние десятилетия дисперсии полимерных частиц, известные как латексы, получили широкое применение при решении задач в областях, где решающую роль играют объемные, коллоидные и поверхностные свойства дисперсного материала. В настоящее время дисперсии частиц преимущественно субмикронного размера начали активно использоваться в высокотехнологичных областях, в частности, для биомедицинских и биотехнологических исследований [1]. Технологии разработки латексов с инновационными свойствами основываются на получении дисперсий частиц, обладающих такими характеристиками, как большая площадь поверхности, гетерогенный полимерный состав, возможность получения частиц разных размеров $(10 \mathrm{~nm}-1 \mathrm{~mm})$ и форм с узкими распределениями по размерам, морфологии, функциональности, а также образования органо-неорганических (гибридных) частиц [2].

Все эти свойства должны тщательно контролироваться и характеризоваться с использованием соответствующих микроскопических методик.

В последнее время многофункциональные полимерные частицы, имеющие несколько функциональных составляющих, например флуоресцентную и магнитную, привлекают большое внимание [3], в первую очередь благодаря их применению в системах мультиплексного анализа, предназначенных для одновременного обнаружения нескольких биомаркеров в одном образце биологического материала (например, сыворотки). Ос- новой мультиплексного анализа является комбинация проточной цитометрии с использованием флуоресцентно окрашенных микросфер, конъюгированных со специфическими антителами. Флуоресцентно меченые магнитные микросферы, применяемые в многопараметрических системах иммунного анализа, кодируются уникальной спектрально отличимой комбинацией красителей и содержат встроенные магнитные наночастицы (МНЧ), что позволяет сформировать в проточной ячейке подобие микрочипов для последующего флуоресцентного анализа присутствия патогена на их поверхности. Таким образом, каждая такая микросфера представляет собой нанотехнологическую ,лабораторию“ для обнаружения биомаркеров [4].

Результаты мультиплексного анализа в одном образце выявляются с помощью двухлазерной системы и цифровой обработки сигналов на проточном флуоресцентном анализаторе [5]. Мультимаркерный анализ позволяет повысить чувствительность и специфичность диагностики и является одним из перспективных подходов к персонификации медицины [6].

Разработка и совершенствование таких нанотехнологических систем мультиплексного анализа, а также контроль их качества невозможны без использования современных методов мультимодальной микроскопии высокого разрешения. Чтобы охарактеризовать все наноструктурные свойства таких систем, необходимо при- 


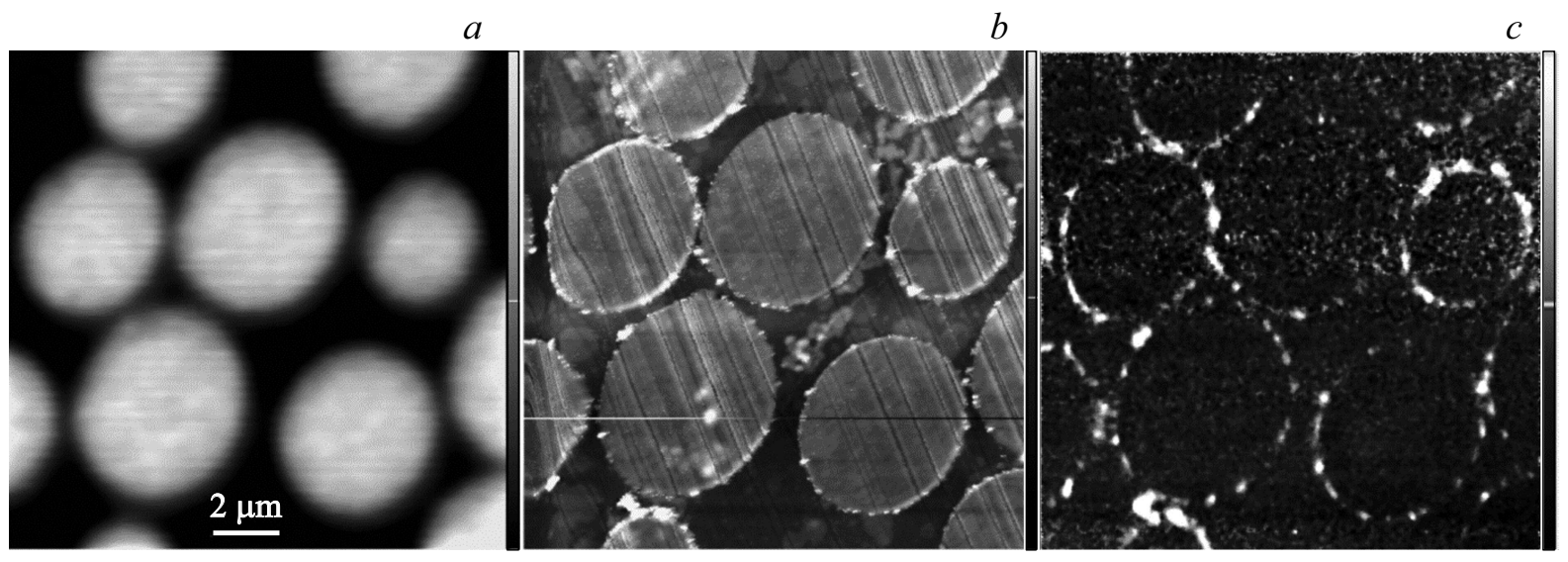

Рис. 1. Исследование образца микросфер Luminex 39 методом СЗНТ-ОМ. $a-$ флуоресцентное конфокальное изображение образца микросфер Luminex 39 в области $655 \mathrm{~nm}$, размер скана $14 \times 14 \mu \mathrm{m} ; b$ - топографическое СЗМ-изображение того же участка; $c$ - МСМ-изображение того же участка.

a

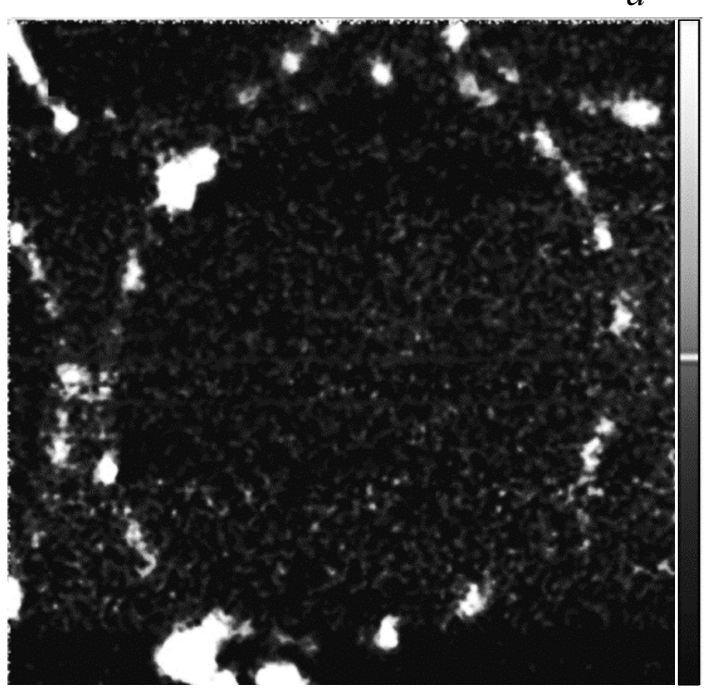

b

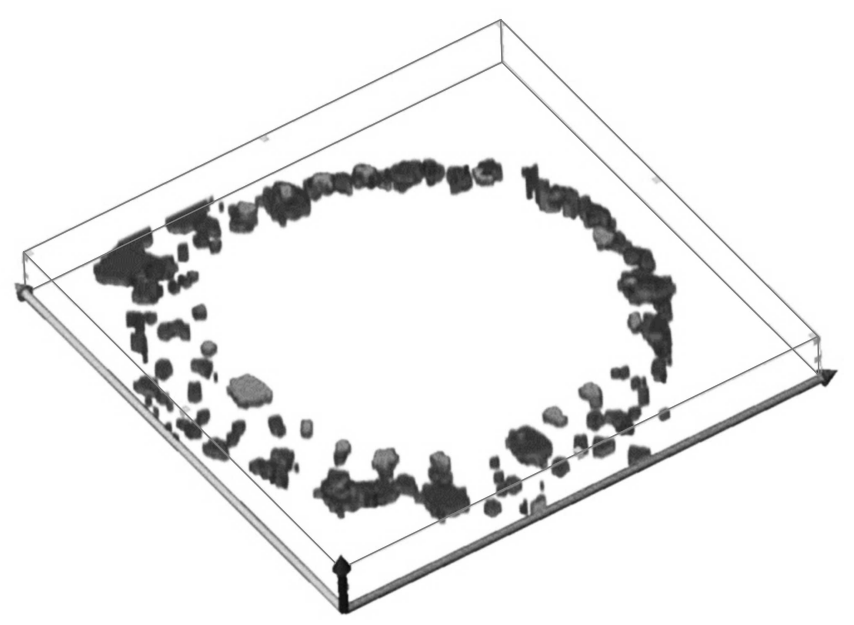

Рис. 2. $a-$ С 3 М-изображение среза микросферы Luminex 39 с увеличенным разрешением, размер скана $7 \times 7 \mu$ m: $b-$ трехмерная реконструкция распределения магнитных наночастиц на поверхности микросферы Luminex 39 , paзмер $7.0 \times 7.0 \times 1.0 \mu$ m, десять срезов толщиной $100 \mathrm{~nm}$.

менять ряд различных устройств и методов. Это делает анализ сложным и трудоемким.

Упомянутые выше трудности, возникающие при характеризации дисперсий полимерных частиц, особенно композитных, гибридных и многофункциональных, приводят к сложной задаче - разработке инструментального подхода, объединяющего возможности различных методов в одном устройстве. Измерение вязкоупругих, флуоресцентных, магнитных свойств с пространственным разрешением является основой для характеризации как гибридных, так и многофункциональных частиц. Сочетание в одном устройстве различных методов, не требующих дополнительных флуоресцентных меток, отвечающих за исследование всех свойств частиц и позволяющих осуществлять внутреннюю трехмерную реконструкцию образца, представляет собой сложную задачу в области характеризации полимерных частиц, что приводит к необходимости разработки уникальных специализированных измерительных техник.

В связи с этим технология сканирующей зондовой нанотомографии-оптической микроскопии (СЗНТ-OM) предоставляет уникальные возможности для анализа наноструктурных особенностей таких микросфер, имеющие решающее значение для обеспечения функциональности систем мультиплексного анализа $[7,8]$. Технология основана на сочетании ультрамикротома, сканирующего зондового микроскопа (СЗМ) и оптического микроспектроскопа в одной системе, предназначенной для последовательных корреляционных измерений поверхности срезаемого образца с помощью ряда зондовых 
и оптических методов в рабочем цикле среза ультрамикротома. Это позволяет производить трехмерную реконструкцию наноструктур в объеме образца путем интеграции последовательных СЗМ- и оптических изображений ряда срезов образца с использованием уникальной научной установки - системы зондово-оптической $3 \mathrm{D}$ корреляционной микроскопии, созданной авторами настоящей работы (http://ckp-rf.ru/usu/486825/). Инструмент входит в состав оборудования ЦКП ИБХ РАН (проект RFMEFI62117X0018).

В настоящей работе исследованы структура и пространственная корреляция расположения области, маркированной флуоресцентным красителем, и области МНЧ внутри микросфер. В качестве объектов для исследований были выбраны микросферы серии Luminex 39 (Luminex Corp., Остин, Техас, США) с включениями МНЧ, используемые на практике в системах мультиплексной диагностики.

Целью исследования является изучение внутренней структуры микросфер, в частности распределения слоев, наночастиц и флуоресцентных красителей в объеме микросфер, а также оценка магнитных свойств обнаруженных наночастиц и спектров флуоресценции микросфер.

Изучение приготовленных образцов микросфер проводилось с помощью атомно-силовой микроскопии $(\mathrm{ACM})$ в полуконтактном режиме и магнитно-силовой микроскопии (МСM) в корреляции с конфокальной флуоресцентной микроскопией и спектроскопией.

Образцы встроенных в эпоксидную среду микросфер срезались с помощью ультрамикротома Reichert-Jung Ultracut (Leica Microsystems GmbH, Вена, Австрия), интегрированного в систему СЗНТ-ОМ, с использованием алмазного ножа UltraAFM 35 (Diatome AG, Биль, Швейцария). Толщина срезов составляла $100 \mathrm{~nm}$. Комбинированная система СЗНТ-ОМ подробно описана в работе [7]. Данная система включает в себя специализированные модули сканирующего зондового микроскопа и оптического микроскопа, позволяющие выполнять как зондовые, так и оптические измерения структур на поверхности образца непосредственно после сверхтонкого среза его поверхности алмазным ножом ультрамикротома без дополнительных перемещений образца. В предположении, что распределения параметров и компонентов в объеме микросфер имеют сферическую симметрию, корреляционные измерения проводились на центральных срезах микросфер, встроенных в эпоксидные блоки.

Поверхность секционированного блока анализировалась с использованием АСМ (топография) и МСМ (распределение намагниченности); та же площадь $(14.0 \times 14.0 \mu \mathrm{m})$ поверхности анализировалась с помощью конфокальной флуоресцентной микроскопии. Магнитные измерения осуществлялись в двухпроходном режиме: сканирование проводилось на первом проходе в полуконтактном режиме АCM, на втором проходе магнитный зонд был поднят на $25 \mathrm{~nm}$ над поверхностью

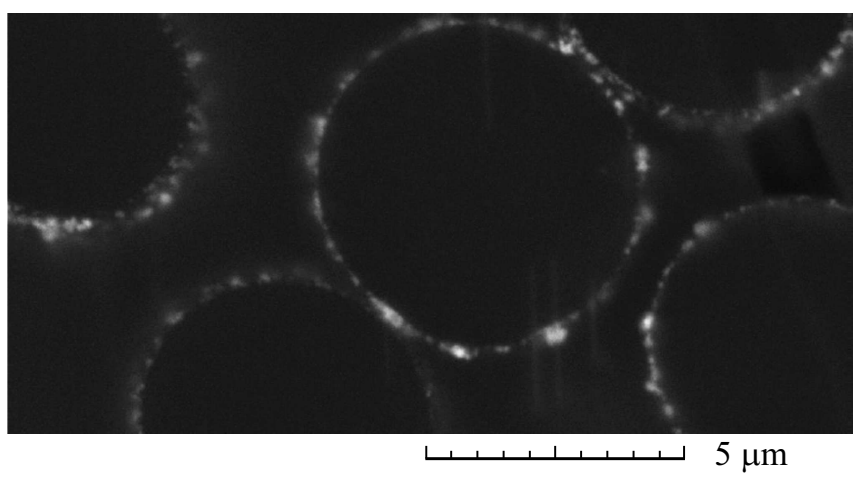

Рис. 3. СЭМ-изображение среза поверхности образца Luminex 39 в режиме обратного рассеяния электронов.

и было получено фазовое изображение, соответствующее картированию распределения намагниченности. Для МСМ-измерений были использованы магнитные кантилеверы ASYMFM (Oxford Instruments Asylum Research) с покрытием зонда $\mathrm{CoCr}$ и резонансной частотой $70 \mathrm{kHz}$. Для АСМ- и МСМ-измерений размер сканирования был установлен на $512 \times 512$ пикселей при частоте сканирования $0.8 \mathrm{~Hz}$.

Чтобы получить трехмерное распределение магнитных свойств, МСМ-измерения затем повторялись 10 раз, каждое после выполнения среза ультрамикротомом толщиной $100 \mathrm{~nm}$. Трехмерная реконструкция распределения намагниченности была визуализирована с помощью программного пакета Image Pro 6.0 Plus с опцией 3D Constructor (Media Cybernetics, Inc., США).

Для получения конфокальных изображений в режиме $\lambda$-сканирования флуоресцентный сигнал интегрировался в диапазоне $\pm 10 \mathrm{~nm}$ от максимума спектра флуоресценции с лазерным возбуждением $632.8 \mathrm{~nm}$ и мощностью $200 \mu \mathrm{W}$. Размер области сканирования $(14 \times 14 \mu \mathrm{m})$ совпадал с областью, исследуемой с помощью СЗМ, при этом в случае конфокальной микроскопии эта область была разделена на $100 \times 100$ пикселей, а время накопления составляло $0.2 \mathrm{~s} / \mathrm{px}$.

Микросферы Luminex 39 мечены двумя флуоресцентными красителями с различной длиной волны и интенсивностью флуоресценции. На рис. 1 показаны флуоресцентное конфокальное изображение в области длин волн $655 \mathrm{~nm}(a)$, а также корреляционные топографические СЗМ-изображения $(b)$ и МСМ-изображения $(c)$ сечения микросфер Luminex 39 на том же участке поверхности. Полученные результаты позволяют сделать вывод, что в данном случае МНЧ располагаются только на поверхности полимерных микросфер, характеризующихся однородной структурой и равномерным распределением флуоресцентных красителей. На топографических СЗМ- и МСМ-изображениях с высоким разрешением (рис. $1, b, c)$ можно четко различить отдельные МНЧ и их кластеры. Необходимо отметить, что не все кластеры, присутствующие на СЗМ-изображении, демонстрируют 
магнитный сигнал соответствующей силы. Это исключает артефакты перекрестных помех С3M/MCM. Размеры отдельных МНЧ, согласно топографическому СЗМизображению высокого разрешения (рис. $2, a$ ), находятся в диапазоне $20-100 \mathrm{~nm}$. На рис. 2, $b$ представлена трехмерная реконструкция распределения магнитных наночастиц на поверхности микросферы Luminex 39, полученная в результате интегрирования десяти последовательных МСМ-изображений срезов образца толщиной $100 \mathrm{~nm}$. Можно видеть, что МНЧ довольно равномерно распределены по сферической поверхности микросфер, хотя некоторые кластеры МНЧ все еще присутствуют. Плотность распределения МНЧ на поверхности микросферы можно оценить на уровне $25 \mu \mathrm{m}^{-2}$.

На рис. 3 показано изображение того же образца микросфер Luminex 39, полученное при помощи сканирующей электронной микроскопии $(\mathrm{CЭM})$ в режиме обратного рассеяния электронов. Данные СЭМ подтверждают результаты СЗМ- и МСМ-измерений, которые указывают на то, что металлосодержащие МНЧ расположены только на поверхности полимерных микросфер.

Таким образом, в работе впервые демонстрируется трехмерная реконструкция распределения наномагнитных свойств в микросферах, соответствующая данным ACM-топографии и конфокальной флуоресцентной микроскопии. Представленные результаты демонстрируют, что методология СЗНТ-ОМ позволяет эффективно изучать трехмерное распределение флуоресцентных красителей и магнитных наночастиц в полимерных микросферах. Исследования различных полимерных, композитных, гибридных и биологических систем показывают, что с помощью режима фазовой визуализации СЗМ можно в то же время обнаружить трехмерное распределение гетерогенных полимерных соединений [9], получить информацию о морфологии трехмерных сетей нанопор [10], оценить локальную эластичность и электрические свойства нанокомпозитных и наногибридных материалов в объеме [11]. С учетом дальнейшего развития этой технологии с использованием всех имеющихся возможностей мультимодальной сканирующей зондовой микроскопии и оптической микроспектроскопии для корреляционного анализа технология СЗНТ-ОМ может стать мощным инструментом для мультимодальной нанохарактеризации различных типов полимерных частиц. Комбинация корреляционного трехмерного СЗМ-, МСМ- и оптического анализа также может быть использована в будущем для изучения локализации биосенсоров на основе МНЧ в анализируемых клетках и тканях с наноразмерным разрешением [12].

\section{Финансирование работы}

Работа выполнена при поддержке Российского научного фонда (проект № 18-19-00718).

\section{Конфликт интересов}

Авторы заявляют, что у них нет конфликта интересов.

\section{Список литературы}

[1] Generalova A.N., Zubov V.P. // Colloids Surf. B. 2018. V. 166. P. 303-322.

[2] Bangs L.B. // Pure Appl. Chem. 1996. V. 68. P. 1873-1879.

[3] Generalova A.N., Chichkov B.N., Khaydukov E.V. // Adv. Colloid Interface Sci. 2017. V. 245. P. 1-19.

[4] Brazhnik K., Sokolova Z., Baryshnikova M., Bilan R., Efimov A., Nabiev I., Sukhanova A. // Nanomedicine: NBM. 2015. V. 11. P. 1065-1075.

[5] Houser B. // Arch. Physiol. Biochem. 2012. V. 118. P. 192 196.

[6] Yu X., Schneiderhan-Marra N., Joos T.O. // Clin. Chem. 2010. V. 56. P. 376-387.

[7] Mochalov K.E., Chistyakov A.A., Solovyeva D.O., Mezin A.V., Oleinikov V.A., Vaskan I.S., Molinari M., Agapov I.I., Nabiev I., Efimov A.E. // Ultramicroscopy. 2017. V. 182. P. $118-123$

[8] Bilan R.S., Krivenkov V.A., Berestovoy M.A., Efimov A., Agapov I.I., Samokhvalov P.S., Nabiev I., Sukhanova A. // ChemPhysChem. 2017. V. 18. P. 970-979.

[9] Alekseev A., Efimov A., Loos J., Matsko N., Syurik J. // Eur. Polym. J. 2014. V. 52. P. 154-165.

[10] Efimov A.E., Moisenovich M.M., Bogush V.G., Agapov I.I. // RSC Adv. 2014. V. 4. P. 60943-60947.

[11] Alekseev A., Efimov A., Lu K., Loos J. // Adv. Mater. 2009. V. 21. P. 4915-4919.

[12] Wu M., Huang S. // Mol. Clin. Oncol. 2017. V. 7. P. 738-746. 